\title{
Рабочие характеристики полупроводниковых лазеров на квантовых ямах в зависимости от ширины волноводной области
}

\author{
(C) 3.Н. Соколова ${ }^{1}$, Н.А. Пихтин ${ }^{1}$, С.О. Слипченко ${ }^{1}$, Л.В. Асрян ${ }^{2}$ \\ ${ }^{1}$ Физико-технический институт им. А.Ф. Иоффе Российской академии наук, \\ 194021 Санкт-Петербург, Россия \\ ${ }^{2}$ Virginia Polytechnic Institute and State University, \\ Blacksburg, VA 24061, USA \\ E-mail: zina.sokolova@mail.ioffe.ru; asryan@vt.edu
}

Поступила в Редакцию 22 июля 2021 г.

В окончательной редакции 2 августа 2021 г.

Принята к публикации 2 августа 2021 г.

\begin{abstract}
Теоретически изучены рабочие характеристики полупроводниковых лазеров на квантовых ямах (КЯ) в зависимости от ширины волноводной области (слоя оптического ограничения - optical confinement layer, OCL). Рассчитаны максимальный модовый коэффициент усиления, фактор оптического ограничения (в КЯ, OCL и эмиттерах), плотность порогового тока, концентрации электронов и дырок (в КЯ и OCL), внутренние оптические потери (в КЯ, OCL и эмиттерах), внутренняя дифференциальная квантовая эффективность, токи стимулированной и спонтанной рекомбинаций, выходная оптическая мощность лазера в зависимости от ширины OCL. Показано, что вплоть до плотностей тока накачки 50 кА/см ${ }^{2}$ мощность излучения рассмотренных лазеров слабо зависит от ширины OCL в диапазоне ширин 1.5-2.8 мкм. Этот результат является важным для создания лазеров с большой яркостью излучения, поскольку в таких лазерах для обеспечения малой расходимости излучения используется широкий волновод. Показано, что при очень высоких плотностях тока накачки мощность излучения как функция ширины OCL имеет максимум.
\end{abstract}

Ключевые слова: квантово-размерный полупроводниковый лазер, гетероструктура, волноводная область.

DOI: 10.21883/FTP.2021.12.51711.9721

\section{1. Введение}

Основой современных полупроводниковых лазеров является двойная гетероструктура с раздельным ограничением [1-8]. Развитие эпитаксиальных технологий роста, таких как молекулярно-пучковая, МОС-гидридная и модифицированная жидкофазная эпитаксии, позволило использовать двумерные области (квантовые ямы) в качестве активной области в структурах, что привело к существенному улучшению их характеристик $[3,4]$. Следующим шагом к совершенствованию гетероструктурных лазеров стало дальнейшее понижение размерности активной области и использование нуль-мерных областей (квантовых точек) вместо квантовых ям [9].

В работе [10] были экспериментально изучены ваттамперные характеристики (ВТАХ) лазеров на квантовых ямах (КЯ), излучающих на длинах волн 1.5-1.6 мкм, и проведено их сравнение для структур с широкими и узкими волноводными областями.

В настоящей работе мы подробно исследуем зависимости рабочих характеристик от ширины волноводной области (слоя оптического ограничения - optical confinement layer, OCL) в планарных торцевых полупроводниковых лазерах с резонатором Фабри-Перо. Активная область лазера состоит из двух КЯ, излучающих на длине волны 1.023 мкм.

Для расчета лазерных характеристик мы используем систему из пяти скоростных уравнений, приведенных в работе [11]. Немгновенность доставки носителей заряда в активную область учитывается посредством введения конечной (не бесконечно высокой) скорости захвата из трехмерной волноводной области (OCL) в двумерную активную область (КЯ) [12].

Транспорт носителей заряда через волноводную область исследован, например, в работах [13-19]. В работах, посвященных транспорту носителей, обычно предполагается мгновенный захват в КЯ. В работе [13], использующей программное обеспечение PICS3D от Crosslight Software для численного моделирования и учитывающей транспорт в лазерной структуре, рассчитанные ВтАХ практически совпадают с экспериментальными ВтАХ из наших работ [20,21].

В наших расчетах транспорт носителей заряда отдельным образом не рассматривается. Однако следует отметить, что использование конечной скорости захвата носителей заряда эффективным образом учитывает и немгновенность транспорта до активной области носителей заряда, инжектированных из эмиттерных областей (cladding layers) в волноводную область. Одним из достоинств такого подхода является простота и прозрачность используемой модели. Совпадение экспериментальной и расчетной ВтАХ в нашей модели может быть достиг-

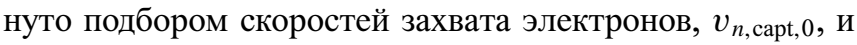

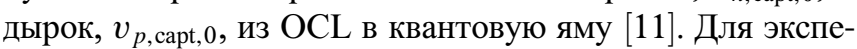
риментальной лазерной структуры, параметры которой приведены в работе [20], наилучшее согласие измеренной [21] и рассчитанной ВтАХ достигается при одинаковых значениях скоростей захвата электронов и дырок 
в пустую КЯ, равных $v_{n, \text { capt, } 0}=v_{p \text {,capt, } 0}=4.3 \cdot 10^{5} \mathrm{~cm} / \mathrm{c}$ (скорости захвата $v_{n \text {, сарt }}$ и $v_{p \text {, сарt }}$, учитывающие заполнение КЯ электронами и дырками, при этом сильно отличаются [22]).

\section{2. Расчет и обсуждение лазерных характеристик}

В настоящей работе мы изучаем влияние ширины OCL планарных полупроводниковых лазерных гетероструктур на их основные характеристики. Теоретически исследуется экспериментальная лазерная структуpa, описание которой приведено в работе [20]; измеренные ВтАХ этой структуры приведены в работах $[20,21]$. Рассматриваются структуры с длиной волны излучения 1.023 мкм с двумя напряженными КЯ InGaAs толщиной $63 \AA$ каждая [20]. КЯ смещены от центра нелегированного OCL, материалом которого является $\mathrm{Al}_{0.1} \mathrm{Ga}_{0.9} \mathrm{As}$. Эмиттеры представляют собой сильно легированные слои (уровень легирования $n^{\text {clad }}=p^{\text {clad }}=5 \cdot 10^{18} \mathrm{~cm}^{-3}$ ) из твердого раствора $\mathrm{Al}_{0.3} \mathrm{Ga}_{0.7} \mathrm{As}$ ( $n$-эмиттер- $n$-cladding layer) и $\mathrm{Al}_{0.6} \mathrm{Ga}_{0.4} \mathrm{As}$ ( $p$-эмиттер- $p$-cladding layer). Длина резонатора ФабриПеро составляет $L=0.3 \mathrm{~cm}$. Ширина полоскового контакта равна 100 мкм, коэффициенты отражения зеркал: $R_{1}=0.05, R_{2}=0.95$. В расчетах ширина OCL $b$ варьируется от 0.5 до 2.8 мкм.

При проведении расчетов мы принимаем во внимание условие глобальной электронейтральности, которое учитывает равенство суммарного заряда электронов в КЯ и OCL суммарному заряду дырок в КЯ и OCL [22]:

$$
e\left(n^{\mathrm{QW}}+b n^{\mathrm{OCL}}\right)=e\left(p^{\mathrm{QW}}+b p^{\mathrm{OCL}}\right)
$$

где $e-$ заряд электрона.

Внутренние оптические потери в нашей модели зависят от тока накачки $j$ (посредством зависимостей концентраций носителей в КЯ и OCL от $j$ ) [23]:

$\alpha_{\text {int }}(j)=\alpha_{0}+\alpha_{\text {int }}^{\mathrm{clad}}+\Gamma^{\mathrm{OCL}} \sigma_{n, \text { int }} n^{\mathrm{OCL}}(j)+\Gamma^{\mathrm{OCL}} \sigma_{p, \text { int }} p^{\mathrm{OCL}}(j)$,

где $\alpha_{0}-$ постоянная компонента внутренних оптических потерь, $\alpha_{\text {int }}^{\text {clad }}-$ внутренние оптические потери в эмиттерах, $\sigma_{n, \text { int }}$ и $\sigma_{p, \text { int }}-$ сечения внутренних оптических потерь на электронах и дырках в OCL, $\Gamma^{\mathrm{OCL}}-$ фактор оптического ограничения в OCL.

Решение системы скоростных уравнений [11] с учетом условия электронейтральности (1) и зависимости внутренних оптических потерь от тока накачки (2) дает нам концентрации электронов и дырок в КЯ $\left(n^{\mathrm{QW}}\right.$ и $\left.p^{\mathrm{QW}}\right)$, в OCL $\left(n^{\mathrm{OCL}}\right.$ и $\left.p^{\mathrm{OCL}}\right)$, а также число фотонов $N$ в лазерной моде резонатора Фабри-Перо в зависимости от ширины OCL $b$ при различных плотностях тока накачки $j$. В расчетах были использованы следующие значения параметров, входящих в (2): $\sigma_{n, \text { int }}=3 \cdot 10^{-18} \mathrm{~cm}^{2}$, $\sigma_{p \text {,int }}=1 \cdot 10^{-17} \mathrm{~cm}^{2}, \alpha_{0}=0.3 \mathrm{~cm}^{-1}$. Температура считалась равной $300 \mathrm{~K}$.

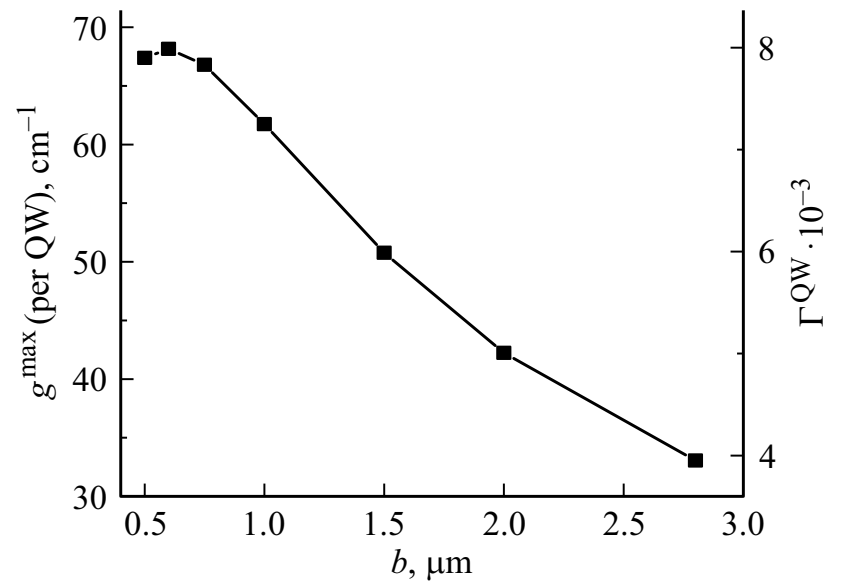

Рис. 1. Зависимость максимального модового коэффициента усиления в одной КЯ (левая ось) и фактора оптического ограничения в одной КЯ (правая ось) от ширины волноводной области (OCL).

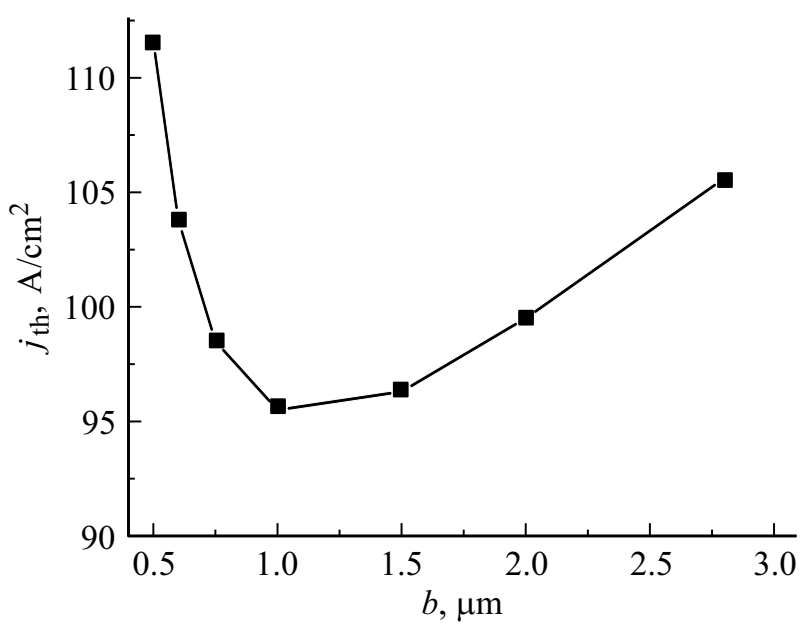

Pис. 2. Плотность порогового тока в зависимости от ширины OCL.

Зависимость максимального модового коэффициента усиления в одной КЯ $\left(g^{\max }\right)$ от ширины OCL приведена на рис. 1 (левая ось). Эта зависимость повторяет зависимость фактора оптического ограничения в одной КЯ $\left(\Gamma^{\mathrm{QW}}\right)$ от ширины OCL (рис. 1, правая ось). Как видно из рисунка, $g^{\max }$ существенно уменьшается с увеличением ширины OCL при значениях $b$, значительно превышающих 0.6 мкм.

На рис. 2 приведена плотность порогового тока $j_{\text {th }}$ в зависимости от ширины OCL. Как видно из рисунка, пороговый ток минимален в структуре с шириной OCL 1 мкм. Существование оптимального значения толщины OCL, минимизирующей плотность порогового тока, обсуждалось в работе [9] в контексте лазеров на квантовых точках.

Зависимость $j_{\text {th }}$ от $b$ повторяет аналогичные зависимости концентраций электронов и дырок в КЯ и OCL на пороге генерации (рис. 3). 

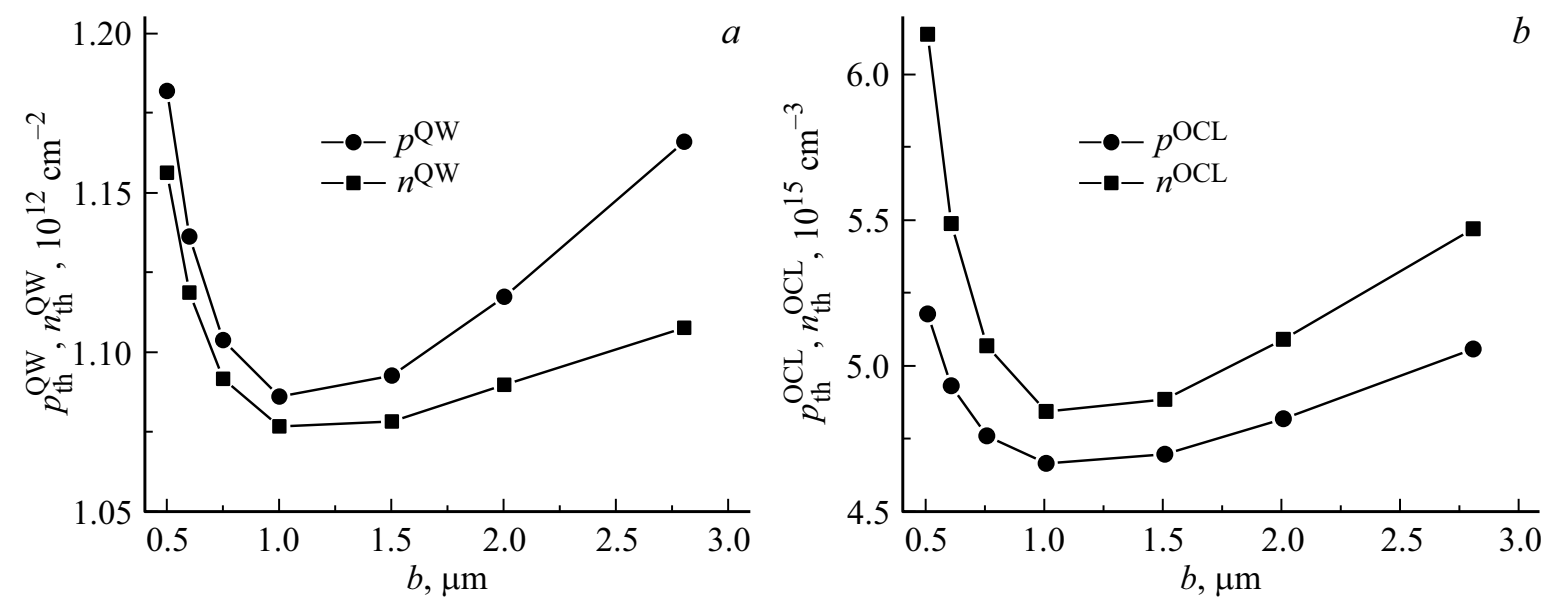

Рис. 3. Пороговые концентрации электронов и дырок в КЯ $(a)$ и OCL $(b)$ в зависимости от ширины OCL.

Выходная оптическая мощность лазера была рассчитана нами в зависимости от ширины OCL $b$ при различных плотностях тока накачки $j[11]$ :

$$
\begin{gathered}
P(b, j)=\hbar \omega c_{g} \beta N(b, j), \\
\beta=\frac{1}{2 L} \ln \frac{1}{R_{1} R_{2}},
\end{gathered}
$$

где $\beta-$ потери, связанные с выходом излучения из резонатора, $\hbar \omega-$ энергия кванта света, $c_{g}-$ групповая скорость света.

Зависимости выходной мощности от ширины OCL лазера при разных плотностях тока накачки приведены на рис. 4. Из рисунка видно, что для любой ширины OCL выходная мощность лазера резко возрастает при увеличении плотности тока накачки вплоть до $70 \mathrm{\kappa A} / \mathrm{cm}^{2}$. Для плотностей тока 15 и 30 кА $/ \mathrm{cm}^{2}$ зависимости $P(b)$ имеют одинаковый характер: при $b>1.5$ мкм выходная мощность практически не зависит от $b$, а при $b<1.5$ мкм с уменьшением ширины OCL выходная мощность резко падает. Для значений $j$ от 50 до $100 \mathrm{\kappa A} / \mathrm{cm}^{2}$ зависимость $P(b)$ имеет максимум, который с увеличением плотности тока смещается в сторону меньшей ширины OCL.

Таким образом, из рис. 4 видно, что вплоть до плотностей тока накачки $50 \mathrm{\kappa A} / \mathrm{cm}^{2}$ мощность излучения рассмотренных лазеров слабо зависит от ширины OCL в диапазоне ширин 1.5-2.8 мкм. Следовательно, можно увеличивать ширину волновода, не уменьшая мощность излучения. Этот результат важен, поскольку использование широких волноводов уменьшает расходимость излучения и дает возможность создавать лазеры с большей яркостью излучения.

Отметим, что для структур с шириной OCL $0.5,0.6$ и 0.75 мкм при очень больших плотностях тока накачки $\left(j>250 \kappa \mathrm{A} / \mathrm{cm}^{2}\right)$ возникает второй порог генерации и вторые ветви в лазерных характеристиках [24].

Падение выходной мощности с уменьшением $b$ при малых значениях $b$ (рис. 4) связано с существенным

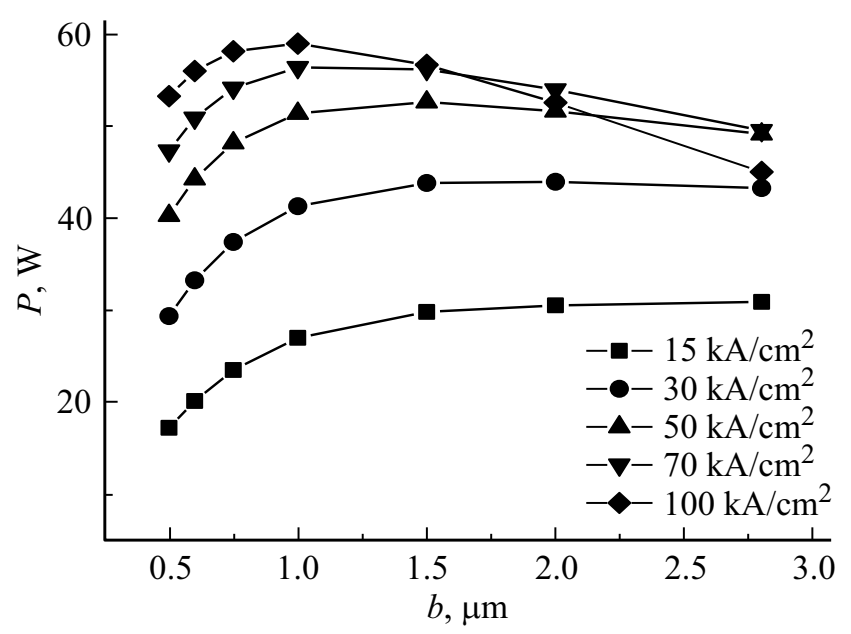

Рис. 4. Зависимость выходной мощности от ширины OCL лазера при разных плотностях тока накачки.

ростом внутренних оптических потерь в $p$-эмиттере при малых значениях ширины OCL (рис. 5,a). Дело в том, что при малых $b$ имеет место значительное проникновение электрического поля в сильно легированный $p$-эмиттер (в исследуемой гетероструктуре активная область смещена к $p$-эмиттеру), что означает существенный рост Г-фактора в $p$-эмиттере (рис. $5, b$ ).

Суммарные внутренние оптические потери, рассчитанные с использованием формулы (2), показаны на рис. 6 в зависимости от ширины OCL при различных плотностях тока накачки. Как видно из рисунка, суммарные потери сильно зависят от плотности тока накачки. При $b<1$ мкм эти потери возрастают с уменьшением ширины OCL, что связано с существенным возрастанием потерь в эмиттерах (рис. 5, $a$ ). Однако при $b>1.5$ мкм даже при очень больших плотностях тока накачки $\left(j \leq 100 \kappa \mathrm{A} / \mathrm{cm}^{2}\right)$ суммарные внутренние оптические потери в структуре слабо зависят от ширины OCL. 

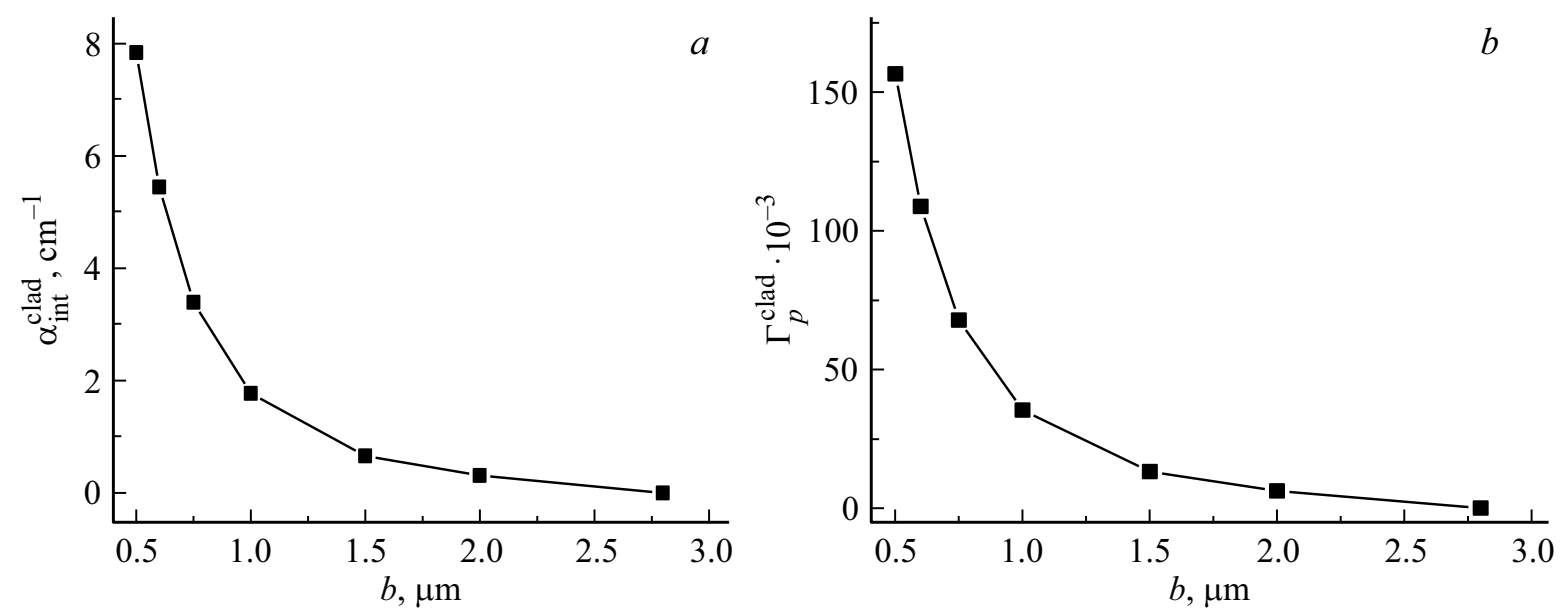

Рис. 5. Зависимость внутренних оптических потерь в эмиттерах $(a)$ и фактора оптического ограничения в $p$-эмиттере $(b)$ от ширины OCL.

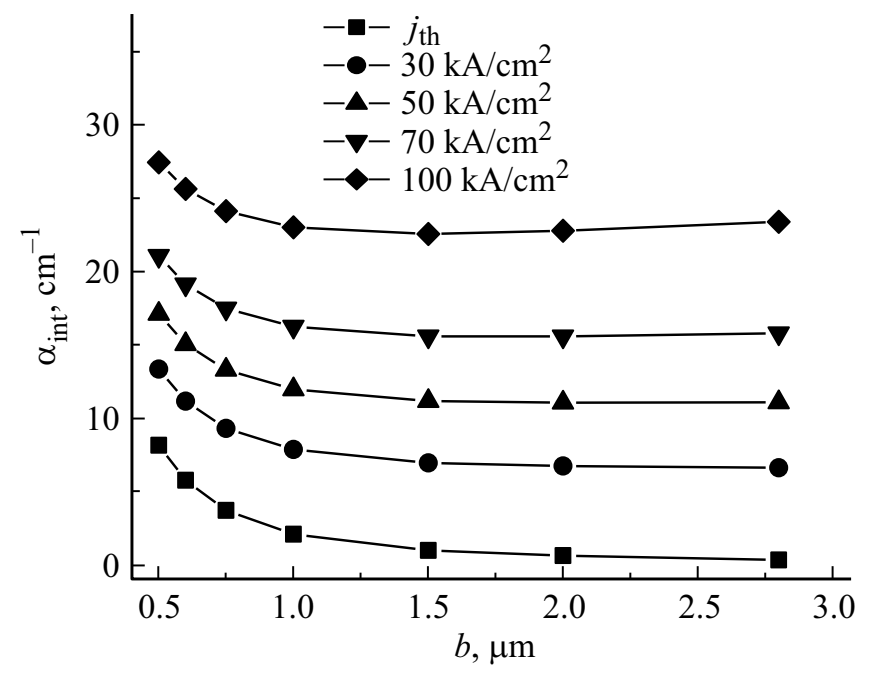

Рис. 6. Зависимость суммарных внутренних оптических потерь в структуре от ширины OCL при разных плотностях тока накачки.

Основной вклад в суммарные внутренние оптические потери вносят потери в OCL $\alpha_{\text {int }}^{\mathrm{OCL}}$ [сумма третьего и четвертого слагаемых в правой части формулы (2)], зависимость которых от $b$ и плотности тока накачки приведена на рис. 7. Наблюдается сильная зависимость от плотности тока накачки и очень слабая от ширины OCL для $j<100 \kappa \mathrm{A} / \mathrm{cm}^{2}$. Как видно из формулы (2), $\alpha_{\mathrm{int}}^{\mathrm{OCL}}$ определяется фактором оптического ограничения в OCL (рис. 8) и концентрациями электронов и дырок в этой области; $n^{\mathrm{OCL}}$ и $p^{\mathrm{OCL}}$ сильно зависят от плотности тока накачки и слабо от $b$ (рис. 9, 10), что и определяет форму кривых для $\alpha_{\text {int }}^{\text {OCL }}$ (рис. 7).

Слабые зависимости внутренних оптических потерь в OCL, а также суммарных потерь в структуре от ширины OCL (рис. 6, 7) не могут быть причиной уменьшения выходной мощности при больших значениях $b$ и $j$ (рис. 4). Уменьшение выходной мощности связано с сильным падением внутренней квантовой эффективности при больших токах накачки, особенно в лазерах с широким OCL (рис. 11). Внутренняя дифференциальная квантовая эффективность (эффективность стимулированного излучения) определяется следующим образом [11]:

$$
\eta_{\text {int }}(b, j)=\frac{j_{\text {stim }}(b, j)}{j-j_{\text {th }}(b)} .
$$

где $j_{\text {stim }}(b, j)$ - плотность тока стимулированной рекомбинации.

Выражение для плотности тока стимулированной рекомбинации можно представить следующим образом [11]:

$$
j_{\text {stim }}(b, j)=e c_{g}\left[\beta+\alpha_{\text {int }}(b, j)\right] \frac{N(b, j)}{S},
$$

где $S$ - площадь полоскового контакта.

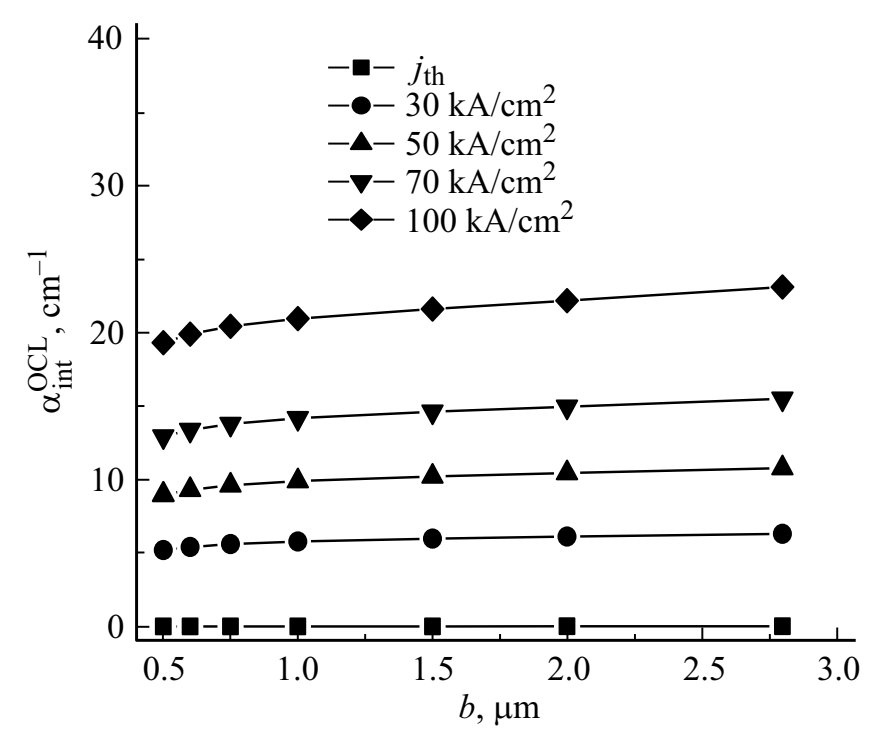

Рис. 7. Зависимость внутренних оптических потерь в OCL от ширины OCL при разных плотностях тока накачки. 


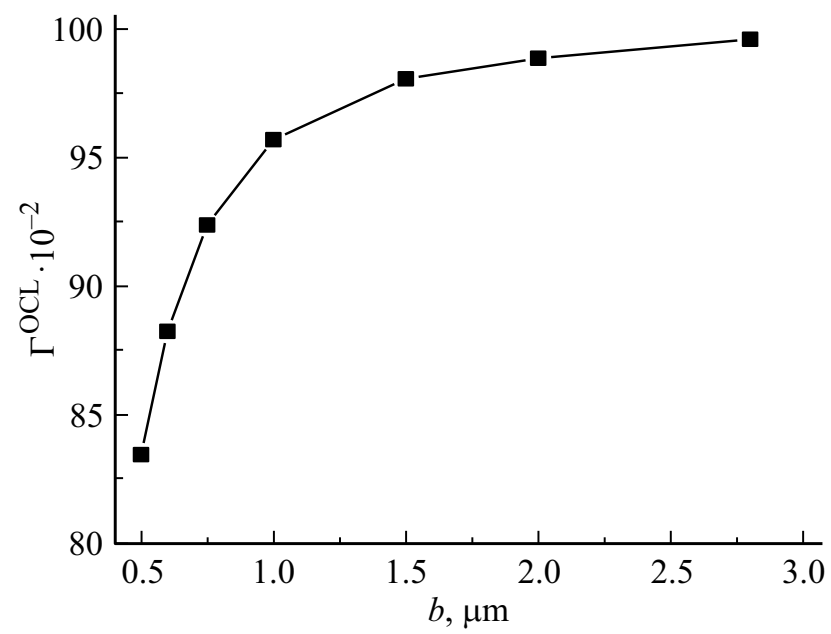

Рис. 8. Зависимость фактора оптического ограничения в OCL от ширины OCL.

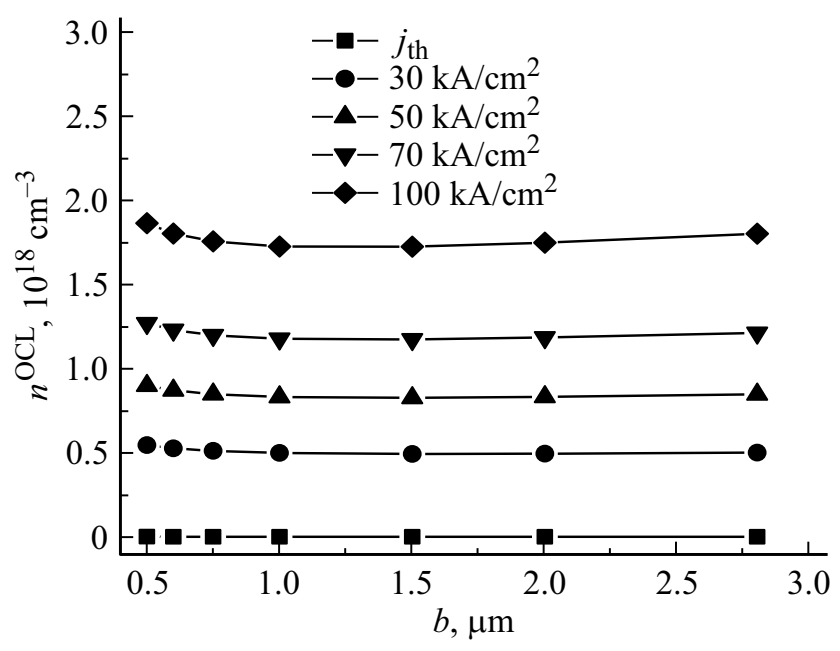

Рис. 9. Зависимость концентрации электронов в OCL от ширины OCL при разных плотностях тока накачки.

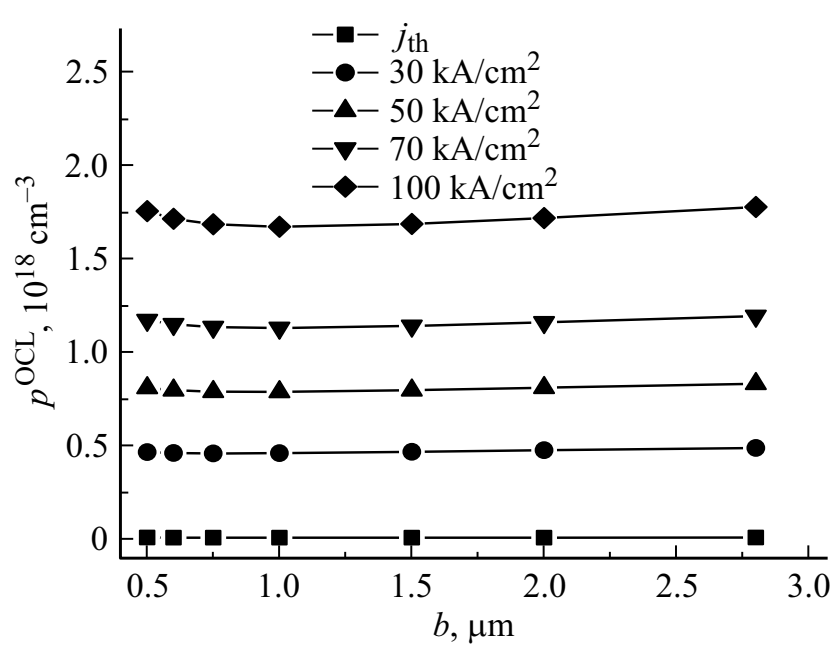

Pис. 10. Зависимость концентрации дырок в OCL от ширины OCL при разных плотностях тока накачки.

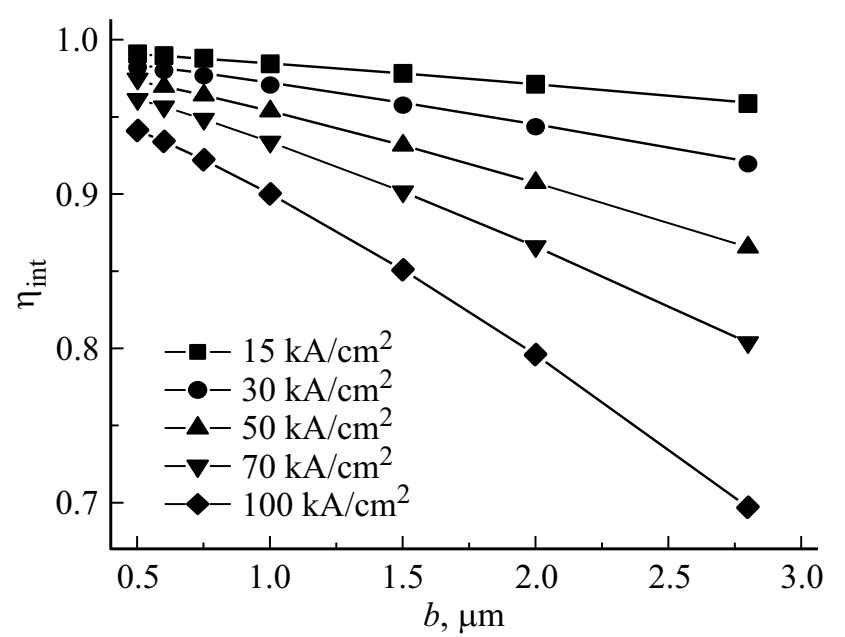

Рис. 11. Зависимость внутренней квантовой эффективности от ширины OCL при разных плотностях тока накачки.

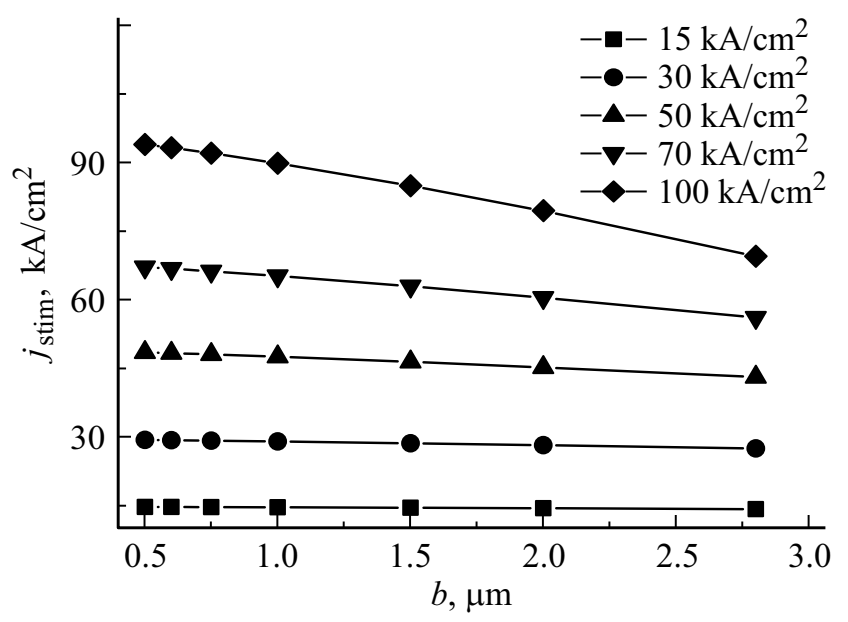

Рис. 12. Зависимость плотности тока стимулированной рекомбинации от ширины OCL при разных плотностях тока накачки.

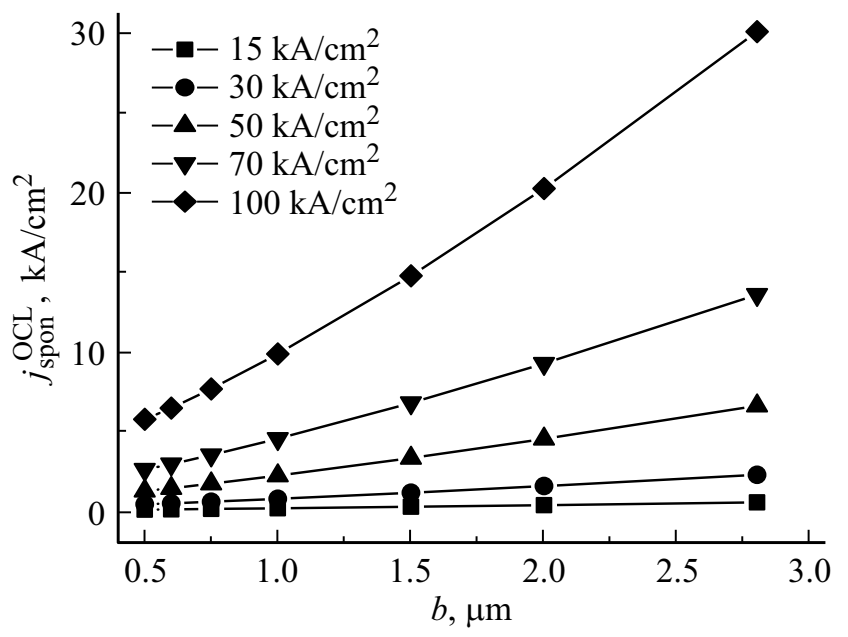

Рис. 13. Зависимость плотности тока спонтанной рекомбинации в OCL от ширины OCL при разных плотностях тока накачки. 
Несмотря на то что плотность тока стимулированной рекомбинации значительно увеличивается при возрастании плотности тока накачки (рис. 12), внутренняя дифференциальная квантовая эффективность при этом падает (рис. 11), особенно в структурах с широким OCL. Это обусловлено возрастанием доли плотности тока накачки, идущей на спонтанную рекомбинацию в OCL. Для плотности тока спонтанной рекомбинации в OCL имеем [11]:

$$
j_{\text {spon }}^{\mathrm{OCL}}(b, j)=e b B^{\mathrm{OCL}} n^{\mathrm{OCL}}(b, j) p^{\mathrm{OCL}}(b, j),
$$

где $B^{\mathrm{OCL}}-$ коэффициент спонтанной излучательной рекомбинации в OCL.

В то время как $j_{\text {spon }}^{\text {OCL }}$ относительно слабо зависит от

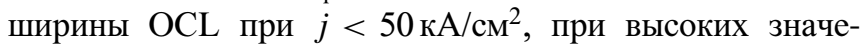
ниях тока накачки $j_{\text {spon }}^{\text {OCL }}$ сильно возрастает с ростом $b$ (рис. 13). Это приводит к значительному уменьшению внутренней квантовой эффективности (рис. 11), тока стимулированной рекомбинации в квантовых ямах (рис. 12) и соответственно к падению выходной мощности лазеров с ростом $b$ при больших токах накачки (рис. 4).

\section{3. Заключение}

Теоретически исследованы лазерные гетероструктуры с шириной волноводной области $b$, изменяющейся в пределах от 0.5 до 2.8 мкм. Показано, что в исследованных структурах при заданной плотности тока накачки, не превышающей 30 кА/ $\mathrm{cm}^{2}$, выходная мощность слабо меняется при изменении ширины OCL от 1.5 до 2.8 мкм. Следовательно, можно увеличивать ширину волновода, не уменьшая мощность излучения. Поскольку использование широких волноводов позволяет уменьшить расходимость излучения, это дает возможность создавать лазеры с большей яркостью излучения.

При $b<1.5$ мкм мощность излучения падает с уменьшением ширины OCL (рис. 4). При плотностях тока накачки, превышающих 40 кА/ $\mathrm{cm}^{2}$, мощность излучения убывает также с увеличением ширины OCL (при $b>1.5$ мкм). Таким образом, при высоких плотностях тока накачки мощность излучения как функция ширины OCL имеет максимум.

Спад выходной мощности при больших значениях ширины OCL и при высоких токах накачки связан с падением внутренней квантовой эффективности (рис. 11). Падение внутренней квантовой эффективности обусловлено возрастанием плотности тока спонтанной рекомбинации в OCL при больших токах инжекции (рис. 13). Следует отметить, что существенная зависимость внутренней квантовой эффективности от ширины OCL начинает проявляться только при высоких плотностях тока накачки $\left(j>30 \kappa \mathrm{A} / \mathrm{cm}^{2}\right)$ (рис. 11$)$.

В то время как суммарные внутренние оптические потери в лазерной структуре сильно зависят от плотности тока накачки, их зависимость от ширины OCL (при $b>1.5$ мкм) оказывается слабой даже при очень высоких плотностях тока накачки (вплоть до $\left.100 \kappa \mathrm{A} / \mathrm{cm}^{2}\right)$. При $b<1$ мкм суммарные внутренние оптические потери возрастают с уменьшением ширины OCL, что связано с существенным возрастанием внутренних потерь на свободных носителях в эмиттерах.

Как и суммарные внутренние оптические потери, внутренние потери в OCL сильно зависят от плотности тока накачки, но слабо зависят от ширины OCL.

\section{Финансирование работы}

Работа выполнена по государственному заданию ФТИ. Л.В. Асрян благодарит Исследовательский офис армии США (U.S. Army Research Office, грант № W911NF-17-1-0432) за поддержку данной работы.

\section{Конфликт интересов}

Авторы заявляют, что у них нет конфликта интересов.

\section{Список литературы}

[1] L. Hayashi. U.S. Patent N 3, 691, 476 (1972).

[2] G. Thompson, P. Kirkby. IEEE J. Quant. Electron., 9 (2), 311 (1973).

[3] W.T. Tsang. Appl. Phys. Lett., 39, 786 (1981).

[4] M.D. Camras, N. Holonyak Jr., M.A. Nixon, R.D. Burnham, W. Streifer, D.R. Scifres, T.L. Paoli, C. Lindström. Appl. Phys. Lett., 42, 761 (1983).

[5] Л.М. Долгинов, А.Е. Дракин, П.Г. Елисеев, Б.Н. Свердлов, В.А. Скрипкин, Е.Г. Шевченко. Квант. электрон., 11 (4), 645 (1984) [Sov. J. Quant. Electron., 14 (4), 439 (1984)].

[6] Ж.И. Алфёров, И.Н. Арсентьев, Д.З. Гарбузов, В.П. Евтихиев, О.В. Сулима, В.П. Чалый, А.В. Чудинов. ФТП, 181 (11), 2057 (1984).

[7] Ж.И. Алфёров, Д.З. Гарбузов, С.В. Зайцев, А.Б. Нивин, А.В. Овчинников, И.С. Тарасов. ФТП, 21 (5), 824 (1987).

[8] Д.З. Гарбузов, А.В. Овчинников, Н.А. Пихтин, 3.Н. Соколова, И.С. Тарасов, В.Б. Халфин. ФТП, 25 (5), 928 (1991).

[9] L.V. Asryan, R.A. Suris. Semicond. Sci. Technol., 11 (4), 554 (1996).

[10] А.А. Мармалюк, Ю.Л. Рябоштан, П.В. Горлачук, М.А. Ладугин, А.А. Падалица, С.О. Слипченко, А.В. Лютецкий, Д.А. Веселов, Н.А. Пихтин. Квант. электрон., 48 (3), 197 (2018). [Quant. Electron., 48 (3), 197 (2018)].

[11] L.V. Asryan, Z.N. Sokolova. J. Appl. Phys., 115, 023107 (2014).

[12] L.V. Asryan, S. Luryi, R.A. Suris. Appl. Phys. Lett., 81, 2154 (2002).

[13] J. Piprek. Optical and Quant. Electron., 51, 60 (2019).

[14] E.A. Avrutin, B.S. Ryvkin. Semicond. Sci. Technol., 32, 015004 (2017).

[15] J. Piprek. Semiconductor Optoelectronic Devices - Introduction to Physics and Simulation (Academic Press, San Diego, 2003).

[16] J. Piprek, Z.M. Li. Photon.Technol. Lett., 30, 963 (2018). 
[17] X. Wang, P. Crump, H. Wenzel, A. Liero, T. Hoffmann, A. Pietrzak, C.M. Schultz, A. Klehr, A. Ginolas, S. Einfeldt, F. Bugge, G. Erbert, G.J. Tränkle. Quant. Electron., 46, 658 (2010).

[18] H. Wenzel, P. Crump, A. Pietrzak, C. Roder, X. Wang, G. Erbert. Optical and Quant. Electron., 41, 645 (2010).

[19] A. Zeghuzi, M. Radziunas, H.-J. Wünsche, A. Klehr, H. Wenzel, A. Knigge. Optical and Quant. Electron., 50, 88 (2018).

[20] Д.А. Веселов, В.А. Капитонов, Н.А. Пихтин, А.В. Лютецкий, Д.Н. Николаев, С.О. Слипченко, 3.Н. Соколова, В.В. Шамахов, И.С. Шашкин, И.С. Тарасов. Квант. электрон., 44 (11), 993 (2014). [Quant. Electron., 44 (11), 993 (2014)].

[21] Д.А. Веселов, Н.А. Пихтин, А.В. Лютецкий, Д.Н. Николаев, С.О. Слипченко, 3.Н. Соколова, В.В. Шамахов, И.С. Шашкин, В.А. Капитонов, И.С. Тарасов. Квант. электрон., $45(7), 597$ (2015). [Quant. Electron., 45(7), 597 (2015)].

[22] Z.N. Sokolova, N.A. Pikhtin, I.S. Tarasov, L.V. Asryan. J. Phys. Conf. Ser., 740, Art. no. 012002 (2016). (5th Int. Symp. on Coherent Optical Radiation of Semiconductor Compounds and Structures, Courses 2015, v. 740, 1 Art No: \#012002, IOP Publishing Issn: 1742-6588, Sept. 2016).

[23] 3.Н. Соколова, Д.А. Веселов, Н.А. Пихтин, И.С. Тарасов, Л.В. Асрян. ФТП, 51 (7), 998 (2017). [Semiconductors, 51 (7), 959 (2017)].

[24] Z.N. Sokolova, N.A. Pikhtin, L.V. Asryan. J. Lightwave Technol., 36 (11), 2295 (2018).

Редактор Л.В. Шаронова

\section{Operating characteristics \\ of semiconductor quantum well lasers \\ as functions of the waveguide region thickness}

Z.N. Sokolova ${ }^{1}$, N.A. Pikhtin ${ }^{1}$, S.O. Slipchenko ${ }^{1}$, L.V. Asryan ${ }^{2}$

${ }^{1}$ loffe Institute, 194021 St. Petersburg, Russia

${ }^{2}$ Virginia Polytechnic Institute and State University, Blacksburg, VA 24061, USA

Abstract Operating characteristics of semiconductor quantum well $(\mathrm{QW})$ lasers are theoretically studied in terms of the thickness of the waveguide region (optical confinement layer, OCL). We calculate the maximum modal gain, optical confinement factor (in QW, OCL, and cladding layers), threshold current density, electron and hole densities (in QW and OCL), internal optical loss (in QW, OCL, and cladding layers), internal differential quantum efficiency, stimulated and spontaneous recombination currents, and output optical power of the laser as functions of the OCL thickness. It is shown that up to the pump current density $50 \mathrm{kA} / \mathrm{cm}^{2}$ the output power of the considered lasers depends only slightly on the OCL thickness in the range of thicknesses $1.5-2.8 \mu \mathrm{m}$. This result is important for designing high brightness lasers as broadened waveguides are used in such lasers to attain low beam divergence. At high pump current densities, the output power is shown to have a maximum as a function of the OCL thickness. 